\title{
Long Term Left Ventricular Reverse Remodeling and Functional Outcome after Cardiac Resynchronization in Advanced Heart Failure
}

Stoskute N, Vaskelyte JJ, Ereminiene E, Puodziukynas A, Kviesulaitis V and Abramaviciute A*

Hospital of Lithuanian University of Health Sciences, Kaunas, Lithuania

"Corresponding author: Agne Abramaviciute, Hospital of Lithuanian University of Health Sciences, Kaunas, Lithuania, Tel: +37064680484; E-mail: abramaviciute.agne@gmail.com

Received date: August 20, 2018; Accepted date: August 31, 2018; Published date: September 05, 2018

Copyright: ( 2018 Stoskute $\mathrm{N}$, et al. This is an open-access article distributed under the terms of the Creative Commons Attribution License, which permits unrestricted use, distribution, and reproduction in any medium, provided the original author and source are credited.

\begin{abstract}
Objectives: The purpose of this study was to assess long-term outcomes of cardiac resynchronization therapy (CRT) on left ventricular reverse remodeling and functional recovery in patients with advanced heart failure and to establish predictors for positive response to cardiac resynchronization.

Methods: The study population consisted of 48 patients (age $68 \pm 8.51$ years, $79.55 \%$ male). Patients underwent transthoracic echocardiography, electrocardiogram, the 6-minute walk test (6MWT) and NYHA functional class evaluation before biventricular pacemaker implantation and during follow-up.

Results: Median follow-up was $21.89 \pm 9.21$ months. $4(7.7 \%)$ patients died from heart failure. Reverse left ventricular remodeling was observed in $77.08 \%(\mathrm{~N}=37)$ patients. CRT resulted in statistically significant improvement of NYHA functional class $(p=0.015)$ and the increased results of 6MWT $(p=0.000)$. Logistic regression analysis demonstrated lower pulmonary artery systolic pressure (PASP) (OR 1.152, 95\% Cl 1.026-1.293; $p=0.017$ ) and more severe mitral regurgitation (OR $0.11,95 \% \mathrm{Cl} 0.018-0.769 ; p=0.025$ ) prior to device implantation as significant predictors of positive response to CRT. Poor response cut-off value of PASP $\geq 46 \mathrm{mmHg}$ was established in ROC analyses, with $78 \%$ of sensitivity and $62 \%$ of specificity.

Conclusions: Our study confirmed the reversible cardiac remodeling and improvement in systolic left and right ventricular function and functional condition in advanced heart failure patients treated with CRT. According to our study, lower pulmonary artery pressure and more severe mitral regurgitation prior to device implantation predicted favorable long-term response to CRT.
\end{abstract}

Keywords: Cardiac resynchronization therapy; Chronic heart failure; CRT responder; Reverse remodeling

\section{Introduction}

The guidelines for chronic heart failure recommend cardiac resynchronization therapy (CRT) as a class I indication, level A evidence, for clinical patients' outcomes improvement. Traditionally several types of indices are used to assess response to CRT: clinical, functional, electrical, echocardiographic, hemodynamic, neurohormonal [1-7].

Despite various studies ascertainment that CRT is efficient for the majority of patients, there are a significant proportion of nonresponders. The literature review demonstrates different criteria of positive response to CRT in clinical trials and rate of non-responders varies from $20 \%$ to $40 \%$.

Accuracy of echocardiographic, electrical, clinical and functional parameters to predict response to CRT remains controversial [8-12].

The purpose of this study is to assess long-term outcomes of CRT on left ventricular reverse remodelling and functional recovery in patients with advanced heart failure and to establish predictors for positive response to cardiac resynchronization therapy.

\section{Materials and Methods}

The study population consisted of 48 patients (age $68.0 \pm 8.5$ years, $79.5 \%$ male) who were treated with CRT-P at the Hospital of Lithuanian University of Health Sciences from 2014/01/01 to 2016/10/31 and met the standard indications for this therapy: New York Heart Association (NYHA) functional class II-IV (ambulatory), LVEF of $35.0 \%$ or less, prolonged QRS interval more than $120.0 \mathrm{~ms}$ and on optimal medical therapy.

The study protocol was approved by the local ethics committee and all subjects provided written informed consent. According to study protocol patients underwent transthoracic 2-dimentional echocardiography (TTE), surface electrocardiogram (ECG), the 6minute walk test (6MWT) and NYHA functional class evaluation before biventricular pacemaker implantation and during follow-up.

According to the response to CRT, patients were classified as responder or non-responder. The response was considered as positive, if left ventricular end-systolic volume index decreased more than $10.0 \%$ during the observation period. The lack of reduction of left ventricular end-systolic index and/or death from heart failure was considered as non-response to CRT.

Depending on primary disease, patients were classified as "ischemic" or "non- ischemic". Those, who have had myocardial 
Page 2 of 6

infarction, angina and coronary artery stenosis more than $75.0 \%$, were considered as "ischemic".

Echocardiographic characteristics including left ventricular enddiastolic diameter and index (LVEDD, LVEDDi), left ventricular endsystolic and end-diastolic volumes and indices (LVESV, LVESVi, LVEDV, LVEDVi), left ventricular ejection fraction (LVEF), grade of left ventricular diastolic dysfunction, left atrial diameter, volume and index (LA, LAV, LAVi), grade of mitral valve regurgitation (MVR), right atrial and right ventricular diameters (RA, RV), tricuspid annular peak systolic excursion (TAPSE), velocity of the tricuspid annular systolic motion (RV S'), pulmonary artery systolic pressure (PASP) were analysed.

Statistical analysis was performed using "Statistical Package of Social Sciences" (SPSS 22.0) and "Microsoft Excel 2010" software. There were defined statistical characteristics, such as total observation number, mean, median and standard deviation by using descriptive statistics. Wilcoxon signed-rank test (for quantitative variables) and McNemar's tests (for qualitative variables) were used to evaluate changeability and statistical reliability of statistical data. Spearman's correlation coefficient ( $r \mathrm{~s})$ was applied to measure the strength and direction of association between two ranked variables. Chi-square (for qualitative variables) and Mann-Whitney $\mathrm{U}$ non-parametric tests (for quantitative variables) were used to assess the relationship between the observation in each group and before and after treatment. Variables with a $p$ value $<0.05$ were considered statistically significant. Receiver operating characteristic (ROC) curve was used to determine a cut-off point of categorical predictors. Variables significant in univariate analysis were added to logistic regression to determine independent predictors of response to CRT. Precision of the model was verified with the Hosmer-Lemeshow test of goodness of fit test.

\section{Results}

Median follow-up was $21.9 \pm 9.2$ months. $4(7.7 \%)$ patients died from heart failure during observation period. The improvement of left ventricular function echocardiographic characteristics during followup was observed in study population: the average LVEF increased, LVEDD, LVEDDi, left ventricular volumes and their indices reduced. MVR grade diminished, moderate or severe MVR was established in 4 pts $(9.1 \%)$ at follow-up vs 17 pts (35.4\%) prior to device implantation $(\mathrm{p}=0.007)$.

The reduction of PASP and increase of TAPSE and RVS revealed the right ventricular function amelioration. Changes of echocardiographic parameters are shown in Table 1.

\begin{tabular}{|l|l|l|l|}
\hline Parameter & Pre-CRT (N=48) & Post-CRT (N=44) & p-value \\
\hline LVEF $(\%)$ & $23.0 \pm 8.2$ & $33.6 \pm 10.8$ & 0 \\
\hline LVEDD $(\mathrm{mm})$ & $62.9 \pm 6.5$ & $59.5 \pm 8.2$ & 0 \\
\hline LVEDDi $\left(\mathrm{mm} / \mathrm{m}^{2}\right)$ & $32.1 \pm 5.0$ & $30.2 \pm 5.6$ & 0.001 \\
\hline LVESV $(\mathrm{ml})$ & $133.7 \pm 47.3$ & $104.3 \pm 68.6$ & 0 \\
\hline LVESVi $\left(\mathrm{ml} / \mathrm{m}^{2}\right)$ & $68.2 \pm 24.9$ & $53.0 \pm 35.2$ & 0 \\
\hline LVEDV $(\mathrm{ml})$ & $172.0 \pm 45.4$ & $151.5 \pm 75.1$ & 0.018 \\
\hline LVEDVi $\left(\mathrm{ml} / \mathrm{m}^{2}\right)$ & $87.7 \pm 24.3$ & $76.7 \pm 38.2$ & 0.016 \\
\hline LA $(\mathrm{mm})$ & $48.0 \pm 5.9$ & $48.0 \pm 6.1$ & $>0.05$ \\
\hline
\end{tabular}

\begin{tabular}{|l|l|l|l|}
\hline LAV $(\mathrm{ml})$ & $95.9 \pm 28.3$ & $107.3 \pm 44.4$ & $>0.05$ \\
\hline LAVi $\left(\mathrm{ml} / \mathrm{m}^{2}\right)$ & $48.6 \pm 14.5$ & $54.0 \pm 21.3$ & $>0.05$ \\
\hline PASP $(\mathrm{mmHg})$ & $42.9 \pm 13.0$ & $34.0 \pm 12.2$ & 0 \\
\hline TAPSE $(\mathrm{mm})$ & $15.6 \pm 3.2$ & $19.0 \pm 4.5$ & 0 \\
\hline RV S'(cm/s) & $9.2 \pm 3.3$ & $10.6 \pm 2.5$ & 0 \\
\hline RV $(\mathrm{mm})$ & $36.0 \pm 4.8$ & $38.2 \pm 6.5$ & 0.02 \\
\hline RA $(\mathrm{mm})$ & $47.0 \pm 6.1$ & $47.3 \pm 7.2$ & $>0.05$ \\
\hline
\end{tabular}

Table 1: Changes of echocardiographic parameters (LVEF: left ventricular ejection fraction; LVEDD: left ventricular end-diastolic diameter; LVEDDi: left ventricular end-diastolic diameter index; LVESV: left ventricular end-systolic volume; LVESVi: left ventricular end-systolic volume index; LVEDV: left ventricular end-diastolic volume; LVEDVi: left ventricular end-diastolic volume index; LA: left atrial diameter; LAV: left atrial volume; LAVi: left atrial volume index; PASP: pulmonary artery systolic pressure; TAPSE: tricuspid annular plane systolic excursion; RVS: velocity of the tricuspid annular systolic motion; RV: right ventricular diameter; RA: right atrial diameter; preCRT: before device implantation; post-CRT: follow up after device implantation).

The significant reduction in left ventricular electrical dyssynchrony comparing follow-up QRS complex duration with prior to device implantation was observed: QRS complex narrowed from $178.7 \pm 21.7$ $\mathrm{ms}$ to $135.3 \pm 16.2 \mathrm{~ms}(\mathrm{p}=0.000)$.

Significant weak correlation between QRS complex duration and left ventricular function echocardiographic characteristics was revealed: LVEF rs=0.40, LVESV rs $=0.35$, LVESVi rs $=0.36$, LVEDV $\mathrm{rs}=0.30$, LVEDVi rs $=0.31$.

Functional parameters, which are representative of the patient's clinical outcome, were evaluated. CRT resulted in statistically significant improvement of NYHA functional class and the increased results of 6MWT (Table 2).

\begin{tabular}{|l|l|l|l|}
\hline Parameter & Pre-CRT (N=48) & Post-CRT (N=44) & p-value \\
\hline \multirow{3}{*}{ NYHA functional class } & $\mathrm{I}-0.0 \%(\mathrm{~N}=0)$ & $\mathrm{I}-18.2 \%(\mathrm{~N}=8)$ & 0.015 \\
\cline { 2 - 4 } & $\mathrm{II}-8.3 \%(\mathrm{~N}=4)$ & $\mathrm{II}-59.1 \%(\mathrm{~N}=26)$ & \\
\cline { 2 - 4 } & $\mathrm{III}-66.7 \%(\mathrm{~N}=32)$ & $\mathrm{III}-18.2 \%(\mathrm{~N}=8)$ & \\
\cline { 2 - 4 } & $\mathrm{IV}-25.0 \%(\mathrm{~N}=12)$ & $\mathrm{IV}-4.5 \%(\mathrm{~N}=2)$ & \\
\hline 6MWT $(\mathrm{m})$ & $232.7 \pm 69.7$ & $360.2 \pm 83.3$ & 0 \\
\hline
\end{tabular}

Table 2: Changes of functional parameters in study population (6MWT: 6 minute walk test; pre-CRT: before device implantation; post-CRT: follow up after device implantation).

Reverse left ventricular remodeling was observed in $77.1 \%(\mathrm{~N}=37)$ patients. According to response to CRT, there was not a statistical significant difference of distribution by mean age $(67.5 \pm 9.0$ vs $71.1 \pm$ 9.1 years, $\mathrm{p}>0.05)$ and follow-up duration $(19.4 \pm 9.1$ vs $22.3 \pm 7.3$ months, $\mathrm{p}>0.05$ ) between responder and non-responder groups. All non-responder group patients were male. 
Citation: Stoskute N, Vaskelyte JJ, Ereminiene E, Puodziukynas A, Kviesulaitis V, et al. (2018) Long Term Left Ventricular Reverse Remodeling and Functional Outcome after Cardiac Resynchronization in Advanced Heart Failure. J Gen Pract 6: 365. doi:10.4172/2329-9126.1000365

Page 3 of 6

Responders to CRT differed from non-responders by the lower level of pulmonary hypertension and better right ventricular function prior to device implantation (Table 3).

\begin{tabular}{|c|c|c|c|c|c|c|}
\hline \multirow{2}{*}{\multicolumn{2}{|c|}{ Parameter }} & \multicolumn{2}{|c|}{ Responder (N=37) } & \multicolumn{2}{|c|}{ Non-responder $(\mathrm{N}=11)$} & \multirow{3}{*}{$\begin{array}{l}\text { p-value } \\
0.838\end{array}$} \\
\hline & & Mean \pm SD & p-value & Mean \pm SD & p-value & \\
\hline \multirow[b]{2}{*}{ LVEF (\%) } & Pre-CRT & $23.4 \pm 8.2$ & \multirow[b]{2}{*}{0.000} & $22.8 \pm 7.3$ & \multirow[b]{2}{*}{0.588} & \\
\hline & Post-CRT & $35.5 \pm 10.0$ & & $23.7 \pm 9.6$ & & 0.016 \\
\hline \multirow[b]{2}{*}{ LVEDD (mm) } & Pre-CRT & $62.1 \pm 6.2$ & \multirow[b]{2}{*}{0.000} & $62.1 \pm 8.9$ & \multirow[b]{2}{*}{0.024} & 0.997 \\
\hline & Post-CRT & $57.4 \pm 6.6$ & & $70.6 \pm 7.0$ & & 0.002 \\
\hline \multirow[b]{2}{*}{ LVEDDi $\left(\mathrm{mm} / \mathrm{m}^{2}\right)$} & Pre-CRT & $32.0 \pm 4.7$ & \multirow[b]{2}{*}{0.000} & $29.9 \pm 6.4$ & \multirow[b]{2}{*}{0.001} & 0.321 \\
\hline & Post-CRT & $29.4 \pm 5.1$ & & $34.2 \pm 7.0$ & & 0.125 \\
\hline \multirow[b]{2}{*}{ LVESV (ml) } & Pre-CRT & $129.9 \pm 48.5$ & \multirow[b]{2}{*}{0.000} & $137.6 \pm 39.1$ & \multirow[b]{2}{*}{0.001} & 0.596 \\
\hline & Post-CRT & $85.2 \pm 46.9$ & & $205.1 \pm 80.0$ & & 0.007 \\
\hline \multirow[b]{2}{*}{ LVESVi $\left(\mathrm{ml} / \mathrm{m}^{2}\right)$} & Pre-CRT & $66.8 \pm 25.0$ & \multirow[b]{2}{*}{0.000} & $66.9 \pm 23.3$ & \multirow[b]{2}{*}{0.000} & 0.991 \\
\hline & Post-CRT & $43.8 \pm 23.4$ & & $101.6 \pm 48.1$ & & 0.018 \\
\hline \multirow[b]{2}{*}{ LVEDV (ml) } & Pre-CRT & $167.7 \pm 46.6$ & \multirow[b]{2}{*}{0.000} & $178.4 \pm 41.4$ & \multirow[b]{2}{*}{0.000} & 0.478 \\
\hline & Post-CRT & $129.2 \pm 49.4$ & & $269.4 \pm 80.6$ & & 0.003 \\
\hline \multirow[b]{2}{*}{ LVEDVi $\left(\mathrm{ml} / \mathrm{m}^{2}\right)$} & Pre-CRT & $86.3 \pm 24.1$ & \multirow[b]{2}{*}{0.000} & $86.2 \pm 24.9$ & \multirow[b]{2}{*}{0.000} & 0.997 \\
\hline & Post-CRT & $66.3 \pm 24.8$ & & $131.8 \pm 50.4$ & & 0.013 \\
\hline \multirow[b]{2}{*}{$\mathrm{LA}(\mathrm{mm})$} & Pre-CRT & $47.4 \pm 5.9$ & \multirow[b]{2}{*}{0.546} & $49.9 \pm 4.7$ & \multirow[b]{2}{*}{0.16} & 0.151 \\
\hline & Post-CRT & $46.9 \pm 6.0$ & & $53.9 \pm 2.3$ & & 0 \\
\hline \multirow[b]{2}{*}{ LAV (ml) } & Pre-CRT & $93.0 \pm 26.4$ & \multirow[b]{2}{*}{0.226} & $927 \pm 38.5$ & & 0.984 \\
\hline & Post-CRT & $101.0 \pm 43.6$ & & $140.7 \pm 34.1$ & 0.053 & 0.022 \\
\hline & Pre-CRT & $44.5 \pm 12.9$ & & $44.8 \pm 21.8$ & & 0.701 \\
\hline LAVi $\left(\mathrm{ml} / \mathrm{m}^{2}\right)$ & Post-CRT & $51.5 \pm 20.9$ & 0.145 & $67.7 \pm 18.8$ & 0.065 & 0.07 \\
\hline & Pre-CRT & $42.0 \pm 12.2$ & & $53.8 \pm 10.2$ & & 0.006 \\
\hline $\operatorname{PASP}(\mathrm{mmHg})$ & Post-CRT & $32.6 \pm 11.0$ & 0.003 & $42.3 \pm 16.0$ & 0.001 & 0.005 \\
\hline & Pre-CRT & $15.7 \pm 3.2$ & & $12.9 \pm 3.7$ & & 0.035 \\
\hline TAPSE (mm) & Post-CRT & $19.2 \pm 4.1$ & 0.026 & $17.9 \pm 6.6$ & 0.045 & 0.636 \\
\hline & Pre-CRT & $9.4 \pm 3.5$ & & $8.2 \pm 1.0$ & & 0.061 \\
\hline$R \vee S^{\prime}(\mathrm{cm} / \mathrm{s})$ & Post-CRT & $10.8 \pm 2.4$ & 0.019 & $9.7 \pm 3.0$ & 0.013 & 0.372 \\
\hline & Pre-CRT & $38.4 \pm 6.6$ & & $38.8 \pm 6.2$ & & 0.85 \\
\hline $\mathrm{RV}(\mathrm{mm})$ & Post-CRT & $37.3 \pm 6.6$ & 0.187 & $43.1 \pm 3.3$ & 0.011 & 0.003 \\
\hline & Pre-CRT & $46.1 \pm 5.6$ & & $49.2 \pm 7.4$ & & 0.218 \\
\hline $\mathrm{RA}(\mathrm{mm})$ & Post-CRT & $46.2 \pm 6.9$ & 0.906 & $53.3 \pm 5.6$ & 0.024 & 0.014 \\
\hline
\end{tabular}

Table 3: Comparison of responders and non-responders echocardiographic parameters before device implantation and at follow-up (LVEF: left ventricular ejection fraction; LVEDD: left ventricular end-diastolic diameter; LVEDDi: left ventricular end-diastolic diameter index; LVESV: left 
ventricular end-systolic volume; LVESVi: left ventricular end-systolic volume index; LVEDV: left ventricular end-diastolic volume; LVEDVi: left ventricular end-diastolic volume index; LA: left atrial diameter; LAV: left atrial volume; LAVi: left atrial volume index; PASP: pulmonary artery systolic pressure; TAPSE: tricuspid annular plane systolic excursion; RVS: velocity of the tricuspid annular systolic motion; RV: right ventricular diameter; RA: right atrial diameter; pre-CRT: before device implantation; post-CRT: follow up after device implantation).

Less prominent electrical dyssynchrony was observed in responders group at follow-up $(\mathrm{p}<0.05)$. QRS duration in responder group narrowed from $175.8 \pm 20.4 \mathrm{~ms}$ to $132.5 \pm 14.3 \mathrm{~ms}(\mathrm{p}<0.005)$, in nonresponder from $187.3 \pm 24.4 \mathrm{~ms}$ to $150.0 \pm 18.9 \mathrm{~ms}(\mathrm{p}<0.005)$.

Significant difference in NYHA functional class distribution of responder and non-responder groups was detected at follow-up, responder's functional condition became better than non-responder. There was $83.8 \%$ (31/37) of NYHA I-II functional class patients in responder group and $42.9 \%(3 / 7)$ in non-responder at follow-up $(\mathrm{p}=0.005)$.

Analysis of echocardiographic parameters at follow-up revealed reduction in left ventricular size, volumes and their indices, better left ventricular systolic and diastolic function, lower degree of mitral regurgitation and lower pulmonary artery systolic pressure in responders group and further progress of left ventricular remodelling and right heart chambers dilatation in non-responders group (Table 3 ).

The redistribution of left ventricular diastolic dysfunction grade at follow-up is demonstrated in Figure 1.

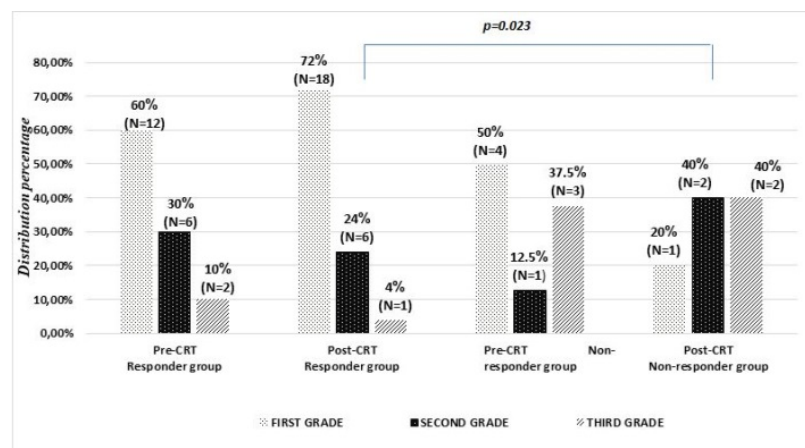

Figure 1: Distribution of LV diastolic dysfunction grade in responder and non-responder groups (LV: left ventricular; pre-CRT: before device implantation; post-CRT: follow-up after device implantation).

The reduction of MVR grade was observed in responder group at follow-up. Moderate or severe MVR was established in 15 pts (40.5\%) prior to device implantation and in 2 pts $(5.4 \%)$ at follow-up period $(p=0.001)$. There wasn't any dynamics of MVR in non-responder group.

According to primary disease, $64.6 \%(\mathrm{~N}=31)$ patients were considered as "ischemic", while $35.4 \%(\mathrm{~N}=17)$ patients "non- ischemic". "Non-ischemic" patients were younger; their PASP was lower and left atrial volume bigger in comparison with "ischemic" patients before device implantation. During follow-up decrease of PASP in both groups patients was observed and difference between groups disappeared. Left atrial volume didn't changed in "non- ischemic" patients group at follow-up, but increased in "ischemic" and exceeds the LAV in "non- ischemic" patients. There were no differences between other analysed parameters according to primary disease. Table 4 summarizes statistically significant differences between "ischemic" and "non- ischemic" patients.

\begin{tabular}{|c|c|c|c|c|c|c|}
\hline \multirow{2}{*}{\multicolumn{2}{|c|}{ Parameter }} & \multicolumn{2}{|l|}{$\begin{array}{l}\text { Ischemic } \\
\mathrm{N}=31\end{array}$} & \multicolumn{2}{|c|}{$\begin{array}{l}\text { Non-ischemic } \\
\mathrm{N}=17\end{array}$} & \multirow{3}{*}{\begin{tabular}{|l}
$\begin{array}{l}\text { p- } \\
\text { value }\end{array}$ \\
0.004
\end{tabular}} \\
\hline & & Mean $\pm S D$ & $\begin{array}{l}\text { p- } \\
\text { value }\end{array}$ & Mean $\pm S D$ & $p$-value & \\
\hline \multicolumn{2}{|c|}{ Age (years) } & $70.8 \pm 9.5$ & - & $63.9 \pm 6.2$ & - & \\
\hline \multirow[b]{2}{*}{$\begin{array}{l}\text { PASP } \\
(\mathrm{mmHg})\end{array}$} & Pre-CRT & $45.7 \pm 13.9$ & & $39.7 \pm 10.6$ & \multirow[b]{2}{*}{0.005} & 0.019 \\
\hline & $\begin{array}{l}\text { Post- } \\
\text { CRT }\end{array}$ & $34.7 \pm 13.1$ & 0.002 & $32.7 \pm 10.7$ & & 0.582 \\
\hline \multirow[b]{2}{*}{ LAV (ml) } & Pre-CRT & $88.7 \pm 31.8$ & & $\begin{array}{l}102.8 \quad \pm \\
20.1\end{array}$ & \multirow[b]{2}{*}{0.145} & 0.07 \\
\hline & $\begin{array}{l}\text { Post- } \\
\text { CRT }\end{array}$ & $111.1 \pm 53.5$ & 0.032 & $\begin{array}{l}100.8 \quad \pm \\
20.7\end{array}$ & & 0.04 \\
\hline
\end{tabular}

Table 4: Differences between "ischemic" and "non-ischemic" patients before device implantation and at follow-up (PASP: pulmonary artery systolic pressure; LAV: left atrial volume; pre-CRT: before device implantation; post-CRT: follow up after device implantation).

Two echocardiographic parameters selected by regression analysis were statistically significant for positive CRT response. It included lower PASP (odds ratio (OR) 1.152, 95.0\%, confidence interval (CI) 1.026-1.293; $\mathrm{p}=0.017)$ and more severe MVR before treatment with CRT (OR 0.11, 95.0\% CI 0.018-0.769; p=0.025). Pre-CRT PASP mean was a significant predictor for positive response with $91.2 \%$ of correct chance. The distribution of PASP means between groups is shown in Figure 2.

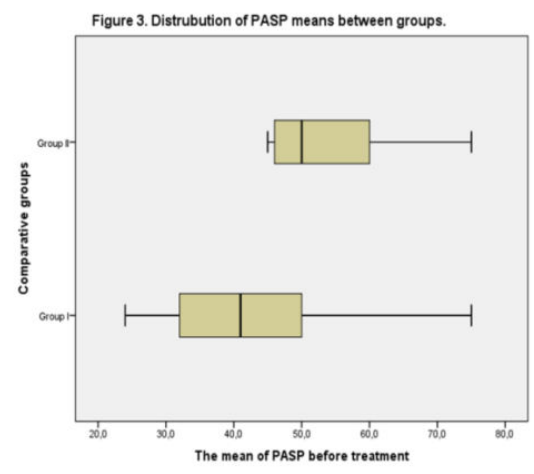

Figure 2: Pre-CRT distribution of PASP means between responder and non-responder groups (Group I responders to CRT, Group II non-responders to CRT). 
Stepwise variable selection with forward selection and backward elimination demonstrated identical results. The precision of the model was verified with the Hosmer-Lemeshow test of goodness of fit test $(\mathrm{p}=0.715)$

Statistically significant cut-off value of PASP was established in ROC analyses, with $78.0 \%$ of sensitivity and $62.0 \%$ of specificity. It demonstrated that PASP $\geq 46.0 \mathrm{mmHg}$ is a predictor of poor response to CRT (Figure 3).

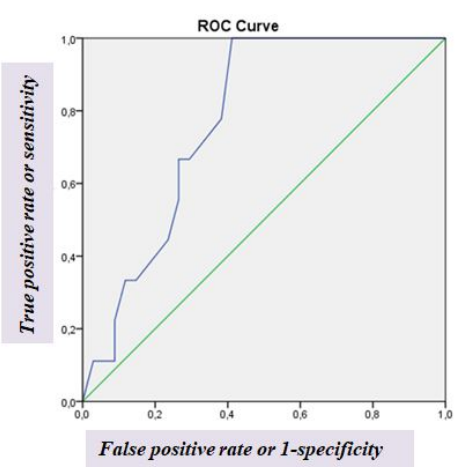

Figure 3: ROC curve for the association of positive response and mean value of PASP (AUC 0.773, $\mathrm{p}=0.013$ ).

\section{Discussion}

The present study confirmed the reversible cardiac remodeling and improvement in systolic left ventricular function in heart failure patients treated with CRT. The significant increase in LVEF and reduction of left ventricular dilatation and grade of MVR revealed. The improvement in right ventricular function characteristics and decrease of pulmonary artery pressure were confirmed. The present findings are similar to previous studies, made by Rapacciuolo et al. and a MIRACLE trial [11-15].

Our study also confirmed that patients treated with CRT achieved significant improvement in their functional condition. We found significant decrease in NYHA functional class and improvement of distance walked in 6-minutes from $232.7 \pm 69.7 \mathrm{~m}$ to $360.2 \pm 83.3 \mathrm{~m}$ $(\mathrm{p}=0.000)$. The miracle trial results were quite similar to ours $[15,16]$. Other clinical trials MUSTIC, CONAK-CD, VENTAC-CHF/ CONTAK-CD results also demonstrated that 6 minute walk distance, quality of life, and NYHA class were significantly improved compared with control group, particularly in the NYHA class III-IV subgroup of patients [15,17].

Several studies have investigated the association between baseline QRS duration and QRS narrowing and reverse left ventricular remodeling during CRT. However, their results have been conflicting. Our current study revealed significant reduction in left ventricular electrical dyssynchrony and weak association between QRS duration and left ventricular volumes and systolic function. Interestingly, the $\Delta$ QRS was very similar in responders and non-responders groups, but electrical dyssynchrony after device implantation was significantly less in responders group $(132.5 \pm 14.3 \mathrm{~ms}$ vs $150.0 \pm 8.9 \mathrm{~ms}, \mathrm{p}=0.007)$. Coppola et al. in their study concluded that patients with a larger decrease in QRS duration after CRT initiation showed greater echocardiographic reverse remodeling [18]. Our findings confirm previous observations of Luparelli et al. that efficiency of CRT depended on the narrowing of QRS complex (127.0 $\pm 17.0 \mathrm{~ms}$ vs 141.0 $\pm 17.0 \mathrm{~ms}, \mathrm{p}<0.05)[10]$.

Our current study shows a $77.1 \%$ prevalence of patients, responding to CRT. This finding is similar to previous studies, where nonresponders proportion ranged from $20 \%$ to $40 \%$ [8-11].

Analysis according to primary disease hasn't revealed ischemic cardiomyopathy as predictor of poor response to CRT as it was shown in previous studies [9]. However, established higher PASP in ischemic patient group and its predictive value capacitate to premise that ischemic patients are less likely to respond to CRT.

Predictors of response to CRT in chronic heart failure patients were analyzed by many clinical studies. CRT response criteria vary between clinical trials, so it is difficult to compare these trials with one another [9]. Possible markers predisposing positive response to CRT were also analyzed in our study. According to our study, lower pulmonary artery pressure and more severe mitral regurgitation prior to device implantation predicted favorable response to CRT. The criteria mentioned above are identified in other studies as well, so it's assumed, that efficiency of CRT can be attributed to multiple factors [9-12, 18-20].

\section{Study Limitations and Future Arrangements}

It is essential to acknowledge that possible limitation of our study is small number of subjects due to the small single-center study design.

Our study future goal is to expand the sample size, in order to assess CRT efficiency predictors more precisely.

\section{Conclusions}

Our study confirmed the reversible cardiac remodelling and improvement in systolic left and right ventricular function and functional condition in advanced heart failure patients treated with CRT. According to our study, lower pulmonary artery pressure and more severe mitral regurgitation prior to device implantation predicted favorable long-term response to CRT.

\section{References}

1. Adelstein E, Saba S (2017) Cardiac resynchronization therapy in heart failure: Indications. Up To Date 3-31.

2. Hawkins NM, Petrie MC, MacDonald MR, Hogg KJ (2006) Selecting patients for cardiac resynchronization therapy: Electrical or mechanical dyssynchrony? Eur Heart J 27: 1270-1281.

3. Daubert JC, Leclercq C, Donal E, Mabo P (2006) Cardiac resynchronisation therapy in heart failure: Current status. Heart Fail Rev 11: $147-154$.

4. Versteeg H, Schiffer AA, Widdershoven JW, Meine MM, Doevendans PA, et al. (2009) Response to cardiac resynchronization therapy: Is it time to expand the criteria? Pacing clinic electrophysiol 32: 1247-1256.

5. Bilchick KC, Lardo AC (2008) Cardiac resynchronization therapy: Application of imaging to optimize patient selection and assess response. Curr Heart Fail Rep 5: 119-127.

6. Lellouche N, De Diego C, Cesario DA, Vaseghi M, Horowitz BN, et al. (2007) Usefulness of preimplantation B-type natriuretic peptide level for predicting response to cardiac resynchronization therapy. Am J Cardiol 99: 242-246.

7. McAlister FA, Ezekowitz J, Hooton N, Vandermeer B, Spooner C, et al. (2007) Cardiac resynchronization therapy for patients with left ventricular systolic dysfunction: A systematic review. JAMA 297: 2502-2514. 
Citation: Stoskute N, Vaskelyte JJ, Ereminiene E, Puodziukynas A, Kviesulaitis V, et al. (2018) Long Term Left Ventricular Reverse Remodeling and Functional Outcome after Cardiac Resynchronization in Advanced Heart Failure. J Gen Pract 6: 365. doi:10.4172/2329-9126.1000365

Page 6 of 6

8. Stahlberg M, Braunschweig F, Gadler F, Mortensen F, Lund LH, et al (2016) Cardiac resynchronization therapy: Results, challenges and perspectives for the future. Scand Cardiovasc J 50: 282-292.

9. Tomassoni G (2016) How to define cardiac resynchronization therapy response. J Innovations in Cardiac Rhythm Management 7: 1-7.

10. Luparelli M, Buccheri D, Corrado E, Ajello L, Bentivegna R, et al. (2016) The importance of being "responder" in cardiac resynchronization therapy. Int J Cardiol 223: 838-841.

11. Rapacciuolo A, Maffe S, Palmisano P, Ferraro A, Cecchetto A, et al. (2016) Prognostic role of right ventricular function in patients with heart failure undergoing cardiac resynchronization therapy. Clin Cardiol 39: 640-645.

12. Witt CT, Kronborg MB, Nohr EA, Mortensen PT, Gerdes C, et al. (2015) Optimization of heart failure medication after cardiac resynchronization therapy and the impact on long-term survival. Eur Heart J Cardiovasc Pharmacothe. 1: 182-188.

13. Praus R, Haman L, Tauchman M, Pudil R, Blaha V, et al. (2012) Echocardiographic changes after cardiac resynchronisation therapy. Kardiologia Polska 70: 1250-1257.

14. Yu CM, Chau E, Sanderseon JE, Fan K, Tang MO, et al. (2002) Tissue Doppler echocardiographic evidence of reverse remodeling and improved synchronicity by simultaneously delaying regional contraction after biventricular pacing therapy in heart failure. Circulation 105: 438-445.
15. Linde C, Ellenbogen K, McAlister FA (2012) Cardiac resynchronization therapy (CRT): Clinical trials, guidelines, and target populations. Heart Rhythm 9: S3-S13.

16. Abraham WT, Fisher WG, Smith AL, Delurgio DB, Leon AR, et al. (2002) Cardiac resynchronization in chronic heart failure. N Engl J Med 346: 19020-1905.

17. Thackray S, Coletta A, Jones P, Dunn A, Clark L, et al. (2001) Clinical trials update: Highlights of the scientific sessions of heart failure 2001, a meeting of the working group on heart failure of the European society of cardiology. CONTAK-CD, CHRISTMAS, OPTIME-CHF. Eur J Heart Fail 3: 491-494.

18. Coppola G, Ciaramitaro G, Stabile G, DOnofrio A, Palmisano P, et al. (2016) Magnitude of QRS duration reduction after biventricular pacing identifies responders to cardiac resynchronization therapy. Int J Cardiol 221: 450-455.

19. Loutfi M, Nawar M, Eltahan S, Elhoda AA (2016) Predictors of response to cardiac resynchronization therapy in chronic heart failure patients. Egypt Heart J 68: 227-236.

20. Spartera M, Galderisi M, Mele D, Cameli M, D'Andrea A, et al. (2016) Role of cardiac dyssynchrony and resynchronization therapy in functional mitral regurgitation. Eur Heart J Cardiovasc Imaging 17: 471-480. 